\title{
A Table showing certain Cultural Characteristics of some of the Commonest Bacteria found in the Labora- tory Tanks at Plymouth.
}

$\mathrm{By}$

\section{G. Harold Drew.}

THE following table is merely intended as a rough classification of eight of the most common species of bacteria found in the Laboratory Tanks. Only the forms which occur free in the water in the greatest numbers have been considered, and the moulds and higher bacteria which can be cultivated from scrapings from the walls of the tanks have not been included.

Until further and more detailed work has been done on the subject, it seemed better to designate the various species by numbers instead of names, since the majority have probably not previously been described.

The culture media employed were similar to those ordinarily used for bacteriological work, with the exception that fish broth (made from dogfish) was substituted for meat broth, and that sea-water was used in place of tap-water. All media were faintly alkaline to Neutral Red.

The medium designated as "Gran's medium," has the following composition :-

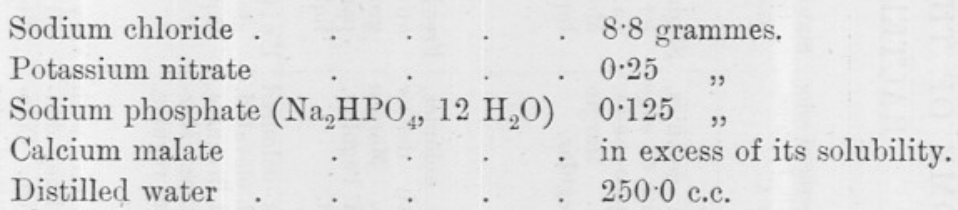

and is fully described by H. H. Gran in the Bergens Museums Aarbog, No. 3, 1901.

The sample of water, from which the bacteria classified in the table were isolated, was collected from just below the surface of one of the tanks in the upper Laboratory at 5.30 p.m. on December 6th, 1909. 0.1 c.c. of this sample plated on Fish-broth, Peptone Agar at $30^{\circ}$ C., gave an average of 130 colonies of all kinds. Samples collected on other occasions show that the eight species described below may be considered as always present and abundant in the tank water. 
TABLE SHOWING SOME OF THE DISTINGUISHING CHARACTERS OF THE COMMONEST BACTERIA IN THE AQUARIUM TANKS.

\begin{tabular}{|c|c|c|}
\hline No & $\begin{array}{c}\text { Morphological charac. } \\
\text { ters of bacteria grown } \\
\text { in Fish broth, Peptone } \\
\text { medium. }\end{array}$ & $\begin{array}{c}\text { Appearance of superficial colo- } \\
\text { nies on Fish broth, Peptone } \\
\text { Agar at } 30^{\circ} \mathrm{C} \text {. }\end{array}$ \\
\hline 1 & $\begin{array}{l}\text { Actively motile rods } \\
\text { with rounded } \\
\text { ends, often ap- } \\
\text { pearing as diplo- } \\
\text { bacilli. Length } \\
\text { of rods about } \\
1 \frac{1}{2} \text { times diam- } \\
\text { eter. }\end{array}$ & $\begin{array}{l}\text { Circular brown colonies, } \\
\text { becoming white at edge } \\
\text { as grow th proceeds. } \\
\text { Slow growth. Margin } \\
\text { smooth and regular. }\end{array}$ \\
\hline 2 & $\begin{array}{l}\text { Actively motile rods } \\
\text { and diplo-bacilli. } \\
\text { Extremely small. }\end{array}$ & $\begin{array}{l}\text { Circular white colonies, } \\
\text { uniform, with denser } \\
\text { spot in centre. Margin } \\
\text { smooth and regular. } \\
\text { Rapid growth. }\end{array}$ \\
\hline 3 & $\begin{array}{l}\text { Small actively mo. } \\
\text { tile vibrio. }\end{array}$ & $\begin{array}{l}\text { Circular cream - coloured } \\
\text { colonies, much elevated. } \\
\text { Brownish spot in centre. } \\
\text { Highly refractive. Mar- } \\
\text { gin smooth and regular. } \\
\text { Rapid growth. }\end{array}$ \\
\hline 4 & $\begin{array}{l}\text { Long spirillum, } \\
\text { actively motile. }\end{array}$ & $\begin{array}{l}\text { Large white colonies with } \\
\text { irregular outline, den- } \\
\text { ser spot in centre. Colo- } \\
\text { nies rapidly spread over } \\
\text { surface. }\end{array}$ \\
\hline
\end{tabular}

\begin{tabular}{|c|c|}
\hline $\begin{array}{l}\text { Stab cultures on Fish } \\
\text { broth, Peptone } \\
\text { Gelatin at } 15^{\circ} \mathrm{C} \text {. }\end{array}$ & $\begin{array}{l}\text { Stab cultures on Fish } \\
\text { broth, Litmus, Lactose, } \\
\text { Peptone Agar at } 30^{\circ} \mathrm{C} \text {. }\end{array}$ \\
\hline $\begin{array}{l}\text { Slight superficial } \\
\text { growth; very } \\
\text { faint growth } \\
\text { along stab. No } \\
\text { liquefaction. }\end{array}$ & $\begin{array}{l}\text { Slight superficial } \\
\text { growth; very } \\
\text { faint growth } \\
\text { along, stab. No } \\
\text { colour change. }\end{array}$ \\
\hline $\begin{array}{l}\text { Free liquefaction on } \\
\text { surface. Free } \\
\text { growth in deeper } \\
\text { parts, but no } \\
\text { liquefaction. }\end{array}$ & $\begin{array}{l}\text { Moderate woolly } \\
\text { looking growth } \\
\text { all along stab. } \\
\text { No colour change. }\end{array}$ \\
\hline $\begin{array}{l}\text { Uniform growth } \\
\text { along stab. No } \\
\text { liquefaction. }\end{array}$ & $\begin{array}{l}\text { Uniform growth } \\
\text { along stab. No } \\
\text { colour change. }\end{array}$ \\
\hline $\begin{array}{l}\text { Very slight growth } \\
\text { all along stab. } \\
\text { No liquefaction. }\end{array}$ & $\begin{array}{l}\text { Very faint growth } \\
\text { near surface. No } \\
\text { colour change. }\end{array}$ \\
\hline
\end{tabular}

\begin{tabular}{|c|c|}
\hline $\begin{array}{l}\text { Stab cultures on Fish } \\
\text { broth, Neutral Red, } \\
\text { Glucose, Peptone } \\
\text { Gelatin at } 15^{\circ} \mathrm{C} \text {. }\end{array}$ & $\begin{array}{l}\text { Denitrification in } \\
\text { Gran's medium } \\
\text { at } 15^{\circ} \mathrm{C} \text {. }\end{array}$ \\
\hline $\begin{array}{l}\text { Slight growth on } \\
\text { surface and to } \\
\text { within } \frac{1}{4} \text { inch be- } \\
\text { low; very faint } \\
\text { growth in deeper } \\
\text { parts. No colour } \\
\text { change. }\end{array}$ & No growth. \\
\hline $\begin{array}{l}\text { Strong growth all } \\
\text { along stab. Li- } \\
\text { quefaction on sur- } \\
\text { face. Acid for- } \\
\text { mation. }\end{array}$ & $\begin{array}{l}\text { Moderate growth. } \\
\text { Nitrite formation } \\
\text { recognizable after } \\
\text { five days. }\end{array}$ \\
\hline $\begin{array}{l}\text { Uniform growth } \\
\text { along stab. No } \\
\text { colour change. } \\
\text { No liquefaction. }\end{array}$ & $\begin{array}{l}\text { Moderate growth, } \\
\text { but no nitrite } \\
\text { formation. }\end{array}$ \\
\hline $\begin{array}{l}\text { Very faint growth } \\
\text { near surface. No } \\
\text { colour change or } \\
\text { liquefaction. }\end{array}$ & $\begin{array}{l}\text { Moderate growth, } \\
\text { but no nitrite } \\
\text { formation. }\end{array}$ \\
\hline
\end{tabular}

Further notes.

Growth in media containing Lactose or Glucose as slightly more rapid than in other media.

Growth in media containing Lac. tose or Glucose was more rapid than in other media.

Presence of Lactose or Glucoseappears to retard growth. 
Small actively motile vibrio.

Small spirillum, non-motile.

Small actively motile rods. motile rods.
Circular white colonies with very granular appearance. Margin hiery irregtlar. Coloabove surface, and of slow growth.

inute actively Large white, clondy-looking colonies, circula line.

White, translucent, highly refractive colonies. Circular. Margin smooth and regular.

Circular white colonies of very rapid growth, showing denser spot in $\mathrm{middle.}$ Uniform smooth margin.

In old cultures the whole medium becomes of golden-brown colour.
Free growth. Funnel-shaped area of liquefaction rapidly spreading downwards.

Growth on surface only. No liquefaction.

Free growth all Free growth all liquefaction at colour change.

first, but after five days it begins to appear.

Rapidly developed funnel-shaped area of liquefaction, soon resulting in the complete liquefaction of all the medium.
Growth all along stab. No colour
change. Woolly appearance.

No growth.
Free growth. Fun- No growth.

nel-shaped lique

faction. No col

our change.

No growth.

Rapid growth, and denitrification apparent in four days.

Free growth all No growth. along stab. No colour change.

Rapid liquefaction with acid formation.

Rapid growth, and denitrification within four days. Ammonia formation apparent at fifth day. 